\title{
ARBEITSSCHUTZ
}

\section{Betriebsarzt und Fachkraft für Arbeitssicherheit unverzichtbar}

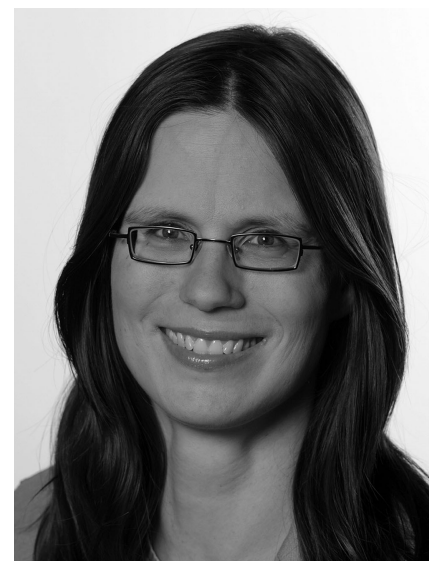

VON SANDRA BIELER Sandra Bieler ist Redakteurin bei der Berufsgenossenschaft für Gesundheitsdienst und Wohlfahrtspflege (BGW) in Hamburg. www.bgw-online.de

\author{
Jeder Betrieb, in dem Arbeitnehmer beschäftigt sind, \\ benötigt eine betriebsärztliche und sicherheitstechnische \\ Betreuung. Das gilt auch für soziale Einrichtungen und \\ Dienste. Je nach Anzahl der Beschäftigten und Situation \\ vor Ort lässt sich diese gesetzlich vorgeschriebene \\ Arbeitsschutzbetreuung unterschiedlich gestalten.
}

Arbeitgeber haben dafür zu sorgen, dass ihre Beschäftigten gesund und sicher arbeiten können. Diese komplexe Aufgabe können sie in der Regel nicht allein erfüllen. Deshalb müssen sie nach dem Arbeitssicherheitsgesetz (ASiG) mit einem Betriebsarzt und einer Fachkraft für Arbeitssicherheit zusammenarbeiten. Diese beraten unter anderem bei der Gefährdungsbeurteilung, der Gestaltung der Arbeitsplätze und Arbeitsabläufe sowie der Auswahl der Persönlichen Schutzausrüstung und des Hautschutzes. Die Fachkraft für Arbeitssicherheit ist der sicherheitstechnische Profi, der Betriebsarzt der medizinische Experte. Er unterstützt den Arbeitgeber beispielsweise auch beim Infektionsschutz, bei der Ersten Hilfe sowie der Wiedereingliederung von Beschäftigten nach längerer Krankheit. Darüber hinaus ist der Betriebsarzt für die arbeitsmedizinischen Vorsorgeuntersuchungen zuständig.

Eigene Betriebsärzte und Sicherheitsfachkräfte zu beschäftigten, lohnt sich nur für große Unternehmen. Ansonsten ist es sinnvoller, auf externe Arbeitsschutzexperten zurückzugreifen. Nach der Unfallverhütungsvorschrift »Betriebsärzte und Fachkräfte für Arbeitssicherheit « (DGUV Vorschrift 2), die die Arbeitsschutzbetreuung seit 2011 regelt, gibt es drei verschiedenen Formen der Betreuung:

- Regelbetreuung für Kleinbetriebe bis zehn Beschäftigte

- Regelbetreuung für Betriebe mit mehr als zehn Beschäftigten

- alternative bedarfsorientierte Betreuung

Welche Betreuungsform für eine Einrichtung oder einen Dienst infrage kommt, hängt unter anderem von der Anzahl der Beschäftigten ab (vgl. Tabelle). Dabei werden Teilzeitkräfte nach einem festen Schlüssel anteilig gezählt: Wer bis zu 20 Stunden pro Woche arbeitet, ist mit dem Faktor 0,5 zu berücksichtigen. Für Teilzeitbeschäftigte mit bis zu 30 Wochenarbeitsstunden gilt der Faktor 0,75. Wer mehr arbeitet, zählt wie eine Vollzeitkraft.

Betrachtet wird bei der Wahl der Betreuungsform grundsätzlich die jeweilige Betriebstätte - also beispielsweise jedes Heim, jeder Pflegedienst, jede Beratungsstelle für sich. Besonders flexibel und eigeninitiativ lässt sich der Arbeitsund Gesundheitsschutz mit der alternativen bedarfsorientierten Betreuung gestalten. Diese wird für Betriebe mit bis zu 50 Beschäftigten angeboten - in der Wohlfahrtspflege derzeit für die Be- 
reiche Pflege, Beratung und Betreuung sowie Kindertagesstätten.

\section{Regelbetreuung für Kleinbetriebe bis zehn Beschäftigte}

Die Regelbetreuung für Kleinbetriebe bis zehn Beschäftigte gliedert sich in eine Grundbetreuung und eine anlassbezogene Betreuung. Erstere beinhaltet hier insbesondere die Unterstützung bei der Gefährdungsbeurteilung. Darüber hinaus ist der Expertenrat in Sachen Arbeitsschutz beispielsweise bei folgenden Anlässen einzuholen:

- Neu- und Umbauten

- wichtige betriebliche Veränderungen

- Erstellung von Notfall- und Alarmplänen

- Gestaltung neuer Arbeitsplätze

- Untersuchung von Unfällen und Berufskrankheiten

Ferner nimmt der Betriebsarzt die arbeitsmedizinischen Vorsorgeuntersuchungen vor.

\section{Regelbetreuung für Betriebe mit mehr als zehn Beschäftigten}

Die Regelbetreuung für Betriebe mit mehr als zehn Beschäftigten umfasst ebenfalls zwei Bausteine: eine Grundbetreuung und eine betriebsspezifische Betreuung.
Berufsgenossenschaft für Gesundheitsdienst und Wohlfahrtspflege empfiehlt, diese jeweils zur Hälfte auf den Betriebsarzt und die Fachkraft für Arbeitssicherheit aufzuteilen.

Die ergänzende betriebsspezifische Betreuung ergibt sich aus dem individuellen Bedarf vor Ort, den die für den

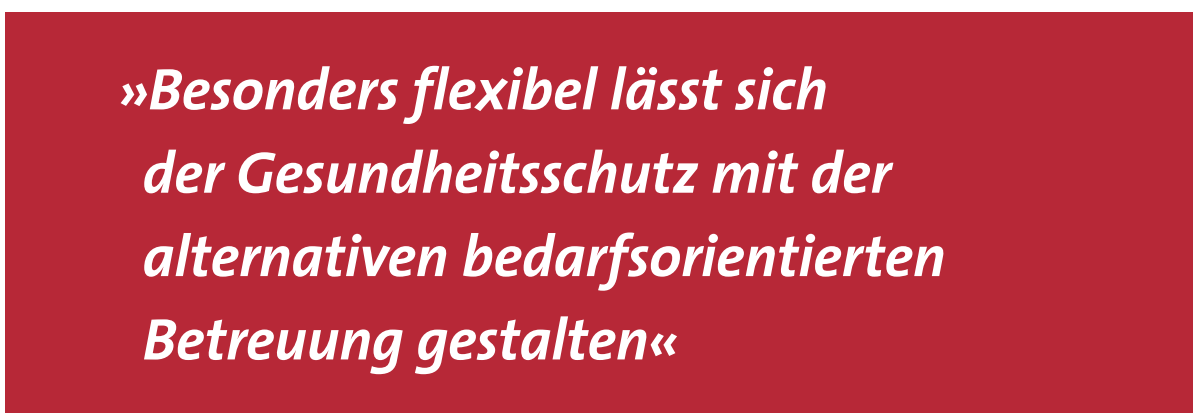

Die Grundbetreuung betrifft hier neben der Gefährdungsbeurteilung unter anderem auch die Arbeitsgestaltung, die Arbeitsorganisation und das Mitwirken in betrieblichen Besprechungen. Für die Grundbetreuung gelten hier feste, einfach zu berechnende Einsatzzeiten. Die

\section{Kooperationspartner und weitere Informationen}

Die Berufsgenossenschaft für Gesundheitsdienst und Wohlfahrtspflege schließt für alle drei Formen der Arbeitsschutzbetreuung Kooperationen mit Dach- und Standesorganisationen, Verbänden sowie betriebsärztlichen und sicherheitstechnischen Dienstleistungsunternehmen - regional und überregional.

Alle Kooperationspartner erfüllen die Qualitätsvoraussetzungen der Berufsgenossenschaft für Gesundheitsdienst und Wohlfahrtspflege und werden unter anderem mit Materialien, Beratung, Erfahrungsaustausch und Oualifizierung von der Berufsgenossenschaft unterstützt. Auch mit einzelnen Verbänden der freien Wohlfahrtspflege arbeitet die Berufsgenossenschaft für Gesundheitsdienst und Wohlfahrtspflege bereits zusammen. Weitere Kooperationen sind möglich.

Die Einrichtungen und Dienste selbst erhalten von der Berufsgenossenschaft für Gesundheitsdienst und Wohlfahrtspflege Informationen und Beratung zur betriebsärztlichen und sicherheitstechnischen Betreuung. Unter www.bgw-online.de, Suche: Arbeitsschutzbetreuung, finden sich unter anderem
- die DGUV Vorschrift 2

- die BGW-Broschüre »Informationen zur DGUV Vorschrift 2. Betriebsärztliche und sicherheitstechnische Betreuungsformen"

- ein Suchassistent zum Ermitteln der möglichen Betreuungsformen

- Musterverträge

- die Liste der Kooperationspartner der Berufsgenossenschaft für Gesundheitsdienst und Wohlfahrtspflege

- eine aktuelle Fragen-und-AntwortenListe

Fragen zur betriebsärztlichen und sicherheitstechnischen Betreuung beantwortet die Berufsgenossenschaft für Gesundheitsdienst und Wohlfahrtspflege unter folgenden Telefonnummern:

Telefon 040 20207-7561 (Sekretariat des Bereichs Betriebsärztliche und sicherheitstechnische Betreuung)

Telefon 080020030330 (Hotline zur betriebsärztlichen und sicherheitstechnischen Betreuung; Anrufe aus dem deutschen Festnetz kostenlos, bei Anrufen aus Mobilfunknetzen können eventuell Kosten entstehen)
Arbeitsschutz zuständige Leitung des Dienstes oder der Einrichtung anhand eines Leistungskatalogs ermittelt. Dabei kann es beispielsweise um die Weiterentwicklung des betrieblichen Gesundheitsmanagements, die Einführung neuer Arbeitsverfahren oder den Umgang mit dem demografischen Wandel gehen. Ferner zählen die arbeitsmedizinischen Vorsorgeuntersuchungen dazu.

Durch die im Jahre 2011 in Kraft getretene DGUV Vorschrift 2 haben sich für die Regelbetreuung für Betriebe mit mehr als zehn Beschäftigten Änderungen ergeben. Sofern noch nicht geschehen, empfiehlt es sich, dass die Einrichtungen und Dienste gemeinsam mit ihrem Betriebsarzt oder ihrer Fachkraft für Arbeitssicherheit klären, ob bestehende Verträge angepasst werden müssen.

\section{Alternative bedarfsorientierte Betreuung}

Bei der alternativen bedarfsorientierten Betreuung lässt sich die Leitung der Einrichtung oder des Dienstes gezielt schulen, um den Arbeits- und Gesundheitsschutz vor Ort möglichst eigenverantwortlich zu gestalten. Voraussetzung ist, dass die verantwortliche Person aktiv in das Betriebsgeschehen eingebunden ist. Sie kümmert sich dann beispielsweise auch eigenständig um die Gefährdungsbeurteilung. Ein Betriebsarzt oder eine Fachkraft für Arbeitssicherheit sind nur bei zusätzlichem Bedarf oder wichtigen Veränderungen im Betrieb hinzuzuziehen. 
Angeboten wird die alternative bedarfsorientierte Betreuung von Dachund Standesorganisation, Verbänden sowie betriebsärztlichen und sicherheitstechnischen Dienstleistungsunternehmen, die mit der Berufsgenossenschaft für Gesundheitsdienst und Wohlfahrtspflege zusammenarbeiten. Diese organisieren unter anderem die Schulungen, für die die Berufsgenossenschaft die Lehrmaterialien zur Verfügung stellt und die Dozenten ausbildet.

Die Berufsgenossenschaft für Gesundheitsdienst und Wohlfahrtspflege steht für alle Fragen der betriebsärztlichen Betreuung und der Arbeitssicherheit als Ansprechpartner zur Verfügung. www.bgw-online.de

\begin{tabular}{|l|l|l|}
\hline $\begin{array}{l}\text { Anzahl der } \\
\text { Beschäftigten }\end{array}$ & Regelbetreuung & $\begin{array}{l}\text { Alternative } \\
\text { bedarfsorientierte } \\
\text { Betreuung }\end{array}$ \\
\hline Bis zu 10 & $\begin{array}{l}\text { Grundbetreuung } \\
\text { + anlassbezogene } \\
\text { Betreuung }\end{array}$ & $\begin{array}{l}\text { Unternehmerschulung } \\
+ \text { bedarfsorientierte } \\
\text { Betreuung }\end{array}$ \\
\hline $\begin{array}{l}\text { Mehr als 10, aber maxi- } \\
\text { mal 50 }\end{array}$ & $\begin{array}{l}\text { Grundbetreuung mit } \\
\text { festen Einsatzzeiten } \\
\text { + betriebspezifische } \\
\text { Betreuung }\end{array}$ & $\begin{array}{l}\text { Unternehmerschulung } \\
\text { + bedarfsorientierte } \\
\text { Betreuung }\end{array}$ \\
\hline Mehr als 50 & $\begin{array}{l}\text { Grundbetreuung mit } \\
\text { festen Einsatzzeiten } \\
+ \text { betriebspezifische } \\
\text { Betreuung }\end{array}$ & Nicht möglich \\
\hline
\end{tabular}

Welche Betreuungsform für eine Einrichtung oder einen Dienst infrage kommt, hängt unter anderem von der Anzahl der Beschäftigten ab: die Betreuungsformen im Überblick

\section{Ideal für Studium und Praxis der Sozialen Arbeit}

\section{Grundsicherung für Arbeitsuchende SGB II}

Herausgegeben von Jens Löcher

2012, 238 S., brosch., 24,- $-€$ ISBN 978-3-8329-5877-0

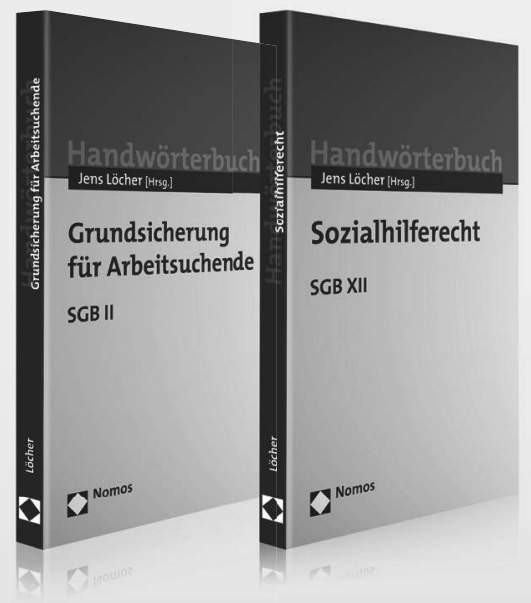

\section{Sozialhilferecht \\ SGB XII \\ Herausgegeben von \\ Jens Löcher}

2013, ca. 250 S., brosch., ca. 24,- $€$ ISBN 978-3-8329-5878-7

Erscheint ca. Dezember 2012
Die Handwörterbücher erfassen die wichtigsten Stichworte aus dem SGB II und SGB XII auf einen Blick. Sozialrechtsberater wie Studierende finden so den schnellen Zugang zu Struktur, Definition wie Relevanz der Begriffe.
Die aktuellen und preiswerten Bände erläutern präzise wie verständlich das Zusammenspiel alter mit neuen Begrifflichkeiten und stellen sie in den praktischen Beratungszusammenhang. Alle Änderungen durch die Hartz-IV-Reform sind berücksichtigt. 